\title{
Estimativa de Atrator de Sistemas Chaveados Obtida Através de Múltiplas Funções Auxiliares
}

\author{
Michele C. Valentino $^{*}, \quad$ Flávio A. Faria ${ }^{\mp}$, \\ *DAMAT- UTFPR, Campus Cornélio Procópio \\ F Instituto de Química, UNESP Univ Estadual Paulista, Campus Araraquara \\ E-mail: valentino@utfpr.edu.br, flaviof15@yahoo.com.br,
}

\author{
Vilma A. Oliveira \\ Universidade de São Paulo, Campus São Carlos \\ E-mail: vilma@sc.usp.br.
}

\begin{abstract}
Resumo: $O$ objetivo deste trabalho é obter uma lei de chaveamento mista, ou seja, uma lei dependente do estado e também do tempo, a qual garante um atrator para o sistema chaveado. $O$ resultado apresentado explora o uso de múltiplas funções auxiliares, as quais desempenham o mesmo papel que as funções de Lyapunov, para estudar o comportamento assintótico das soluções chaveadas e suas principais características são que o resultado pode ser aplicado mesmo quando nem todos os subsistemas são ultimamente limitados e a derivada das funções auxiliares podem assumir valores positivos.
\end{abstract}

Palavras-chave: Sistemas Chaveados, Atratores, Múltiplas Funções Auxiliares

\section{Introdução}

Sistemas chaveados surgem na prática na modelagem de operação de muitos sistemas de engenharia $[1,6]$. Embora chaveamento não seja um novo conceito em engenharia, nas últimas décadas a teoria de sistemas chaveados tem atraído a atenção de muitos pesquisadores. Como consequência, resultados para a estabilidade e estabilização para essa classe de sistemas foi significativamente desenvolvida.

Apesar dos importantes avanços na teoria de estabilidade, os atratores de muitos sistemas chaveados podem não ser um ponto de equilíbrio. Um exemplo clássico é o sistema de controle de temperatura liga-desliga. Para essa classe de problemas, o interesse não é estudar a estabilidade de um ponto particular de equilíbrio mais o comportamento assintótico das soluções.

O princípio de invariância é uma poderosa ferramenta para analisar o comportamento assintótico das soluções de sistemas dinâmicos. Este resultado baseia-se na existência de uma função do tipo Lyapunov para analisar o comportamento assintótico das soluções do sistema. Uma propriedade chave dessa função, a não-positividade de sua derivada ao longo das soluções do sistema chaveado [3]. Encontrar uma função, satisfazendo todas as suposições do princípio de invariância pode ser difícil para muitos sistemas dinâmicos. Portanto, uma extensão do princípio de invariância foi apresentado em [8]. Os resultados foram obtidos através de uma função auxiliar comum e também múltiplas funções auxiliares, as quais desempenham o mesmo papel que as funções de Lyapunov. Apesar de ser permitido que a derivada de tais funções assumam valores positivos em alguns conjuntos, os resultados são somente aplicados quando todos os subsistemas são ultimamente limitados. Para superar esta dificuldade, em [9, 10] as propriedades da função auxiliar $V$ foram analisadas ao longo da solução de um sistema auxiliar formado pela combinação convexa de todos os subsistemas para estudar o comportamento assintótico da solução do sistema chaveado e então as estimativas de atratores puderam ser obtidas mesmo quando nem todos os 
subsistemas são ultimamente limitados. O objetivo do presente trabalho é explorar múltiplas funções de Lyapunov para obter uma lei de chaveamento que garanta um atrator para o sistemas chaveados composto por subsistemas que não precisam ser ultimamente limitados. Então, o resultado será aplicado para uma classe maior de sistemas do que nos resultados obtidos em $[8,9,10]$.

\section{Conceitos Fundamentais}

Considera-se a família $\mathcal{F}=\left\{f_{p}\right\}_{p \in \mathcal{P}}$ de campos vetoriais de classe $C^{1}$, completos do $\mathbb{R}^{n}$ e a seguinte classe de sistemas chaveados contínuos no tempo

$$
\dot{x}(t)=f_{\sigma(.)}(x(t)), \quad x(0)=x_{0}
$$

em que $\mathcal{P}=\{1,2, \ldots, N\}$ é um conjunto de índices finito, $N$ é o número de subsistemas, $x(t) \in \mathbb{R}^{n}$ é o vetor do estado, $\sigma$ é uma função constante por partes chamada de lei de chaveamento, a qual pode depender do tempo $\sigma: I \rightarrow \mathcal{P}$ em que $I=\left[0, t_{f}\right)$ com $0<t_{f} \leq \infty$, ou do estado $\sigma: X \rightarrow P \operatorname{com} X \subseteq \mathbb{R}^{n}$. Uma função contínua suave por partes $x_{\sigma(.)}(t): I \rightarrow \mathbb{R}^{n}$ é uma solução do sistema chaveado (1) no intervalo $I$ se $x_{\sigma(.)}(t)$ satisfaz $\dot{x}_{\sigma(.)}(t)=f_{p}\left(x_{\sigma(.)}(t)\right)$ para todo $t$ tal que $\sigma()=$.$p . Denota-se \varphi_{\sigma(.)}\left(t, x_{0}\right)$, ou simplesmente $\varphi\left(t, x_{0}\right)$, a solução de (1) iniciando em $x_{0}$ no tempo $t=0$ através da lei de chaveamento $\sigma($.$) .$

Neste trabalho, estuda-se soluções do sistema (1) através de uma classe particular de lei de chaveamento, então algumas definições preliminares e proposições [3, 4, 7] as quais serão usadas no desenvolvimento do resultado principal são apresentadas.

Definio 2.1. Seja $H$ um conjunto fechado e invariante para o sistema (1) através da lei de chaveamento $\sigma$. Diz-se que $H$ é um atrator se existe uma vizinhança $U$ de $H$ tal que, para toda condição inicial $x_{0} \in U, \varphi\left(t, x_{0}\right) \rightarrow H$ quando $t \rightarrow+\infty$.

Definio 2.2. (Invariância Fraca) Um conjunto $\mathcal{N}$ é fracamente invariante com respeito ao sistema chaveado (1) se para cada $x_{0} \in \mathcal{N}$ existe um indice $p \in \mathcal{P}$, uma solução $\varphi\left(t, x_{0}\right)$ do campo vetorial $f_{p}(x)$ e um número real $b>0$ tal que $\varphi\left(t, x_{0}\right)$ pertence ao conjunto $\mathcal{N}$ para qualquer $t \in[-b, 0]$ ou $t \in[0, b]$.

Definio 2.3. (Pontos Limites) Um ponto $q \in \mathbb{R}^{n}$ é um ponto limite de $\varphi\left(t, x_{0}\right)$ se existe uma sequência de tempos de chaveamentos $\left\{t_{k}\right\}_{k \in N}$, com $t_{k} \rightarrow \infty$, quando $k \rightarrow \infty$ tal que $\lim _{k \rightarrow \infty} \varphi\left(t_{k}, x_{0}\right)=q$. O conjunto de todos os pontos limites de $\varphi\left(t, x_{0}\right)$ é denotado por $\omega\left(x_{0}\right)$.

Definio 2.4. (Dwell-Time) Diz-se que uma lei de chaveamento é dwell-time se existe uma sequência de tempos de chaveamentos $\left\{\tau_{k}\right\}_{k \in \mathbb{N}}$ e $T>0$ tal que

$$
\inf _{k}\left(\tau_{k+1}-\tau_{k}\right) \geq T \text {. }
$$

O número $T$ é chamado de tempo de permanência para $\varphi\left(t, x_{0}\right)$ e o conjunto de todas as soluções possuindo um chaveamento dwell-time é denotado por $\mathcal{S}_{\text {dwell }} \subset \mathcal{S}$ e essas soluções são chamadas de soluções dwell-time.

Definio 2.5. (Matrizes de Matzler) Matrizes $\Pi \in \mathbb{R}^{n \times n}$ tais que

$$
\pi_{j p} \geq 0, \forall j \neq p, \quad \sum_{j=1}^{N} \pi_{j p}=0, \forall p \in \mathcal{P},
$$

são chamadas de matrizes de Metzler.

Proposio 2.1. (Propriedades de Conjunto Limite) Seja $\varphi\left(t, x_{0}\right) \in \mathcal{S}_{\text {dwell }}$ uma solução chaveada limitada de (1) para $t \geq 0$. Então, $\omega\left(x_{0}\right)$ é não vazio, compacto e fracamente invariante. Além disso, $\varphi\left(t, x_{0}\right)$ é atraído para $\omega\left(x_{0}\right)$. 
Demonstração. Veja [2].

Na próxima seção, é apresentado o resultado principal, o qual fornece estimativas de conjuntos atratores de sistemas chaveados mesmo quando nem todos os subsistemas são ultimamente limitados. O resultado é obtido através de múltiplas funções auxiliares e a lei de chaveamento que garante essa estimativa depende do tempo e também do estado.

\section{Estimativa de Conjuntos Atratores de Sistemas Chaveados Obtida Através de Múltiplas Funções Auxiliares}

Considere $V_{p}: \mathbb{R}^{n} \rightarrow \mathbb{R}$ funções de classe $C^{1}$ para todo $p \in \mathcal{P}$, o conjunto

$$
C=\left\{x \in \mathbb{R}^{n}: \nabla V_{p}(x) \cdot f_{p}(x)+\sum_{j=1}^{N} \pi_{j p} V_{p}(x)<0, \forall p \in \mathcal{P}\right\}
$$

e a seguinte suposição, a qual é necessária para se obter o resultado principal.

Suposio 3.1. Existem funções contínuas $a, b: \mathbb{R}^{n} \rightarrow \mathbb{R}$ satisfazendo $a(x) \leq \inf _{p \in \mathcal{P}} V_{p}$ e $b(x) \geq$ $\sup _{p \in \mathcal{P}} V_{p}(x)$ para todo $x \in \mathbb{R}^{n}$.

Através da Suposição 1, defina $C^{c}$ como o conjunto complementar de (3), $\Omega_{\ell_{0}}=\left\{x \in \mathbb{R}^{n}\right.$ : $\left.a(x) \leq \ell_{0}\right\}, \Omega_{\ell_{1}}=\left\{x \in \mathbb{R}^{n}: a(x) \leq \ell_{1}\right\}$ e $\Theta=\left\{x \in \mathbb{R}^{n}: b(x) \leq \ell_{0}\right\}$, com $\sup _{x \in C^{c}} b(x) \leq \ell_{0}<\infty \mathrm{e}$ $\sup _{x \in \Omega_{\ell_{0}}} b(x) \leq \ell_{1}<\infty$. É claro por construção que

$$
C^{c} \subseteq \Theta \subset \Omega_{\ell_{0}} \subset \Omega_{\ell_{1}}
$$

Observao 3.1. A construção dos conjuntos $\Omega_{\ell_{0}}, \Omega_{\ell_{1}}$ e $\Theta$ segue a estratégia apresentada em [8]. Entretanto neste artigo não há necessidade de construir $N+1$ conjuntos como em [8], com $N$ o número de subsistemas.

A próxima proposição fornece uma lei de chaveamento dependente do estado, a qual garante que a derivada da função $v(x)=\min _{p \in \mathcal{P}} V_{p}(x)$ decresce ao longo da solução do sistema chaveado (1) e é usada na demonstração do resultado principal.

Proposio 3.1. Sejam $\mathcal{B}$ um subconjunto do $\mathbb{R}^{n}, V_{p}: \mathbb{R}^{n} \rightarrow \mathbb{R}$ funções de classe $\mathcal{C}^{1}$ e $\pi_{j p}$ elementos da matriz de Matzler, se para todo $x \in \mathcal{B}$

$$
\nabla V_{p}(x) \cdot f_{p}(x)+\sum_{j=1}^{N} \pi_{j p} V_{p}<0, \forall p \in \mathcal{P}
$$

então, a lei de chaveamento

$$
\sigma(x)=\arg \min _{p \in \mathcal{P}} V_{p}(x)
$$

faz com que a função $v(x)=\min _{p \in \mathcal{P}} V_{p}(x)$ decresa ao longo da solução do sistema chaveado. Mesmo que o chaveamento rápido ocorra, a função $v$ ainda decresce ao longo da solução de Filippov de (1).

Demonstração. Veja [5]. 
No próximo teorema é apresentado o resultado principal deste trabalho e considera-se que $\mathcal{B}=\overline{\Omega_{\ell_{0}}^{c}}$. A lei de chaveamento desse resultado depende do estado fora do conjunto $\mathcal{B}$ e do tempo dentro desse conjunto.

Teorema 3.1. Considere o sistema chaveado (1) e $V_{p}(x): \mathbb{R}^{n} \rightarrow \mathbb{R}$ funções de classe $\mathcal{C}^{1}$ para todo $p \in \mathcal{P}$. Através da Suposição (1), se $\Omega_{\ell_{1}}$ é limitado, então toda solução $\varphi\left(t, x_{0}\right)$ de (1) possuindo uma lei de chaveamento dwell-time em $\Omega_{\ell_{0}}$ e uma lei de chaveamento satisfazendo (5) para todo $x \in \overline{\Omega_{\ell_{0}}^{c}}$ é atraída para um conjunto fracamente invariante em $\Omega_{\ell_{1}}$. Portanto, para qualquer condição inicial $x_{0}$, a solução é atraída para um conjunto fracamente invariante em $\Omega_{\ell_{1}}$ e então, $\Omega_{\ell_{1}}$ é uma estimativa do conjunto atrator do sistema chaveado (1).

Demonstração. Seja $\left\{\tau_{k}\right\}_{k \in \mathbb{N}}$ uma sequência de tempos de chaveamentos. Primeiramente, considere $x_{0} \in \Theta$ e $\varphi\left(t, x_{0}\right)$ sendo uma solução de (1) possuindo um chaveamento dwell-time em $\Omega_{\ell_{0}}$ e uma lei de chaveamento satisfazendo (5) para todo $x \in \overline{\Omega_{\ell_{0}}^{c}}$. Enquanto não ocorre o chaveamento, ou seja, temos um único subsistema $p$ ativado, a solução $\varphi\left(t, x_{0}\right) \in \Omega_{\ell_{0}}$ para todo $t \in\left[\tau_{0}, \tau_{1}\right)$, pois caso contrário, existiria um $\bar{\tau}$ de forma que $V_{p}\left(x_{0}\right) \leq \ell_{0}$ e $V_{p}\left(\varphi\left(\bar{\tau}, x_{0}\right)\right)>\ell_{0}$ e então a função contínua $V_{p}$ cresceria fora de $\Omega_{\ell_{0}}$, mas pela Proposição (2) a lei de chaveamento (5) não permite que isso acontea. No próximo instante de chaveamento a solução pode deixar $\Omega_{\ell_{0}}$, mas não deixará $\Omega_{\ell_{1}}$, pois para todo $x \in \Omega_{\ell_{0}}, V_{p}(x) \leq \ell_{1}$ para todo $p \in \mathcal{P}$. Ainda mais, $v\left(t, x_{0}\right) \leq \ell_{1}$ para todo $t>\tau_{1}$, pois a lei de chaveamento (5) é satisfeita fora de $\Omega_{\ell_{0}}$. Portanto, a solução $\varphi\left(t, x_{0}\right)$ com $x_{0} \in \Theta$ permanece em $\Omega_{\ell_{1}}$ que é limitado e então, pela Proposição 1 a solução é atraída para um conjunto fracamente invariante em $\Omega_{\ell_{1}}$.

Agora, considere $x_{0} \notin \Theta$ e $\varphi\left(t, x_{0}\right)$ sendo uma solução de (1) através de uma lei satisfazendo (5). Então pela Proposição 2, a função $v(x)=\min _{p \in \mathcal{P}} V_{p}(x)$ decresce ao longo da solução de (1) mesmo que o chaveamento rápido ocorra. Portanto existe um tempo $t_{1}$ tal que $v\left(x\left(t_{1}\right)\right)<\ell_{0} \mathrm{e}$ então o resultado segue da primeira parte desta demonstração.

Portanto, o conjunto limitado $\Omega_{\ell_{1}}$ é a estimativa de um conjunto atrator do sistema chaveado, pois para qualquer condição inicial $x_{0}$ a solução é atraída para um conjunto fracamente invariante em $\Omega_{\ell_{1}}$.

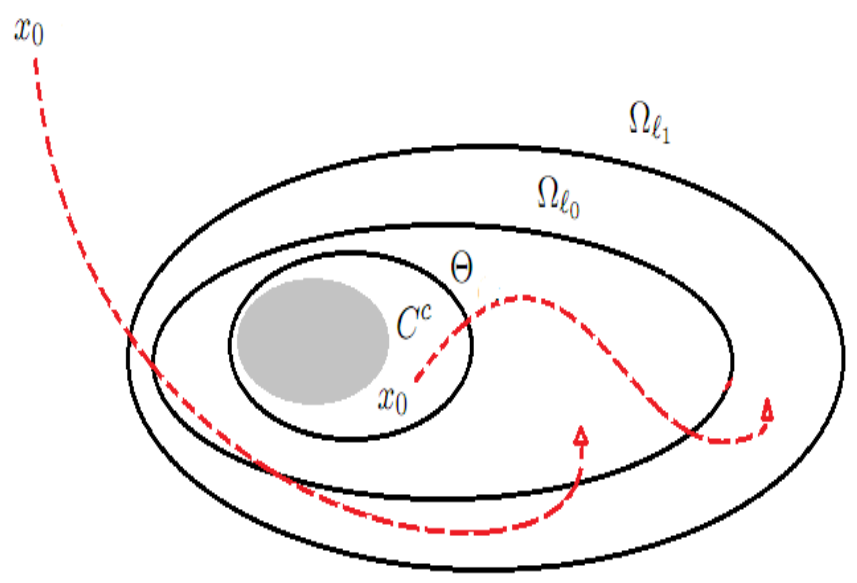

Figura 1: Interpretação geométrica do Teorema 1

Exemplo 3.1. Considere um sistema chaveado com $\mathcal{P}=\{1,2\}$ e

$$
f_{1}(x)=\left[\begin{array}{c}
-x_{1}^{3}+x_{1}+x_{2} \\
-2 x_{2} x_{1}^{2}-x_{2}^{3}+\frac{1}{2} x_{2}+x_{1}
\end{array}\right], \quad f_{2}(x)=\left[\begin{array}{c}
-e^{x_{1}}\left(x_{1}^{3}+x_{1} x_{2}^{2}-x_{1}\right)+x_{2} \\
\frac{x_{2}}{2}-e^{x_{1}}\left(x_{1}^{2} x_{2}+x_{2}^{3}-x_{2}\right)-x_{1}
\end{array}\right]
$$


Seja $V_{1}(x)=\frac{x_{1}^{2}+x_{2}^{2}}{2}, V_{2}(x)=\frac{x_{1}^{2}+2 x_{2}^{2}}{2}, \pi_{11}=\pi_{22}=-1$ e $\pi_{12}=\pi_{21}=1$. Então

$$
\begin{aligned}
& \nabla V_{1}(x) f_{1}(x)+\left(\pi_{11} V_{1}+\pi_{21} V_{2}\right)=\left(x_{1}^{2}+x_{2}^{2}\right)\left(-x_{1}^{2}-x_{2}^{2}+1\right) \\
& \nabla V_{2}(x) f_{2}(x)+\left(\pi_{12} V_{1}+\pi_{22} V_{2}\right)=e^{x_{1}}\left(x_{1}^{2}+x_{2}^{2}\right)\left(-x_{1}^{2}-x_{2}^{2}+1\right) .
\end{aligned}
$$

Portanto, $C^{c}=\left\{x \in \mathbb{R}^{2}: x_{1}^{2}+x_{2}^{2} \leq 1\right\}$. Escolhendo $a(x)=\frac{x_{1}^{2}+x_{2}^{2}}{2}$ e $b(x)=\frac{x_{1}^{2}+2 x_{2}^{2}}{2}$, tem-se

$$
\begin{array}{r}
\ell_{0}=1, \quad \Theta=\left\{x \in \mathbb{R}^{2}: \frac{x_{1}^{2}+2 x_{2}^{2}}{2} \leq 1\right\}, \\
\Omega_{\ell_{0}}=\left\{x \in \mathbb{R}^{2}: \frac{x_{1}^{2}+x_{2}^{2}}{2} \leq 1\right\}, \\
\ell_{1}=2, \quad \Omega_{\ell_{1}}=\left\{x \in \mathbb{R}^{2}: \frac{x_{1}^{2}+x_{2}^{2}}{2} \leq 2\right\} .
\end{array}
$$

Então, pelo Teorema 3.1 qualquer solução $\varphi\left(t, x_{0}\right)$ possuindo uma lei de chaveamento dwell-time em $\Omega_{\ell_{0}}$ e uma lei satisfazendo (5) para todo $x \in \overline{\Omega_{\ell_{0}}^{c}}$ é atraída para um conjunto fracamente invariante em $\Omega_{\ell_{1}}$. A Figura 2(a) ilustra a simulação no domínio de tempo para $x_{0}=[42]$, a Figura 2(b) confirma o resultado do Teorema 3.1 por mostrar um conjunto atrator dentro do círculo de raio 2 para $\ell_{1}=2$. A Figura 3(a) mostra que a função $V_{p}$ não cresce fora de $\Omega_{\ell_{1}} e$ a Figura 3(b) mostra a lei de chaveamento $\sigma($.$) , a qual permite o chaveamento rápido fora do$ conjunto $\Omega_{\ell_{0}}$ pois satisfaz (5) neste conjunto.
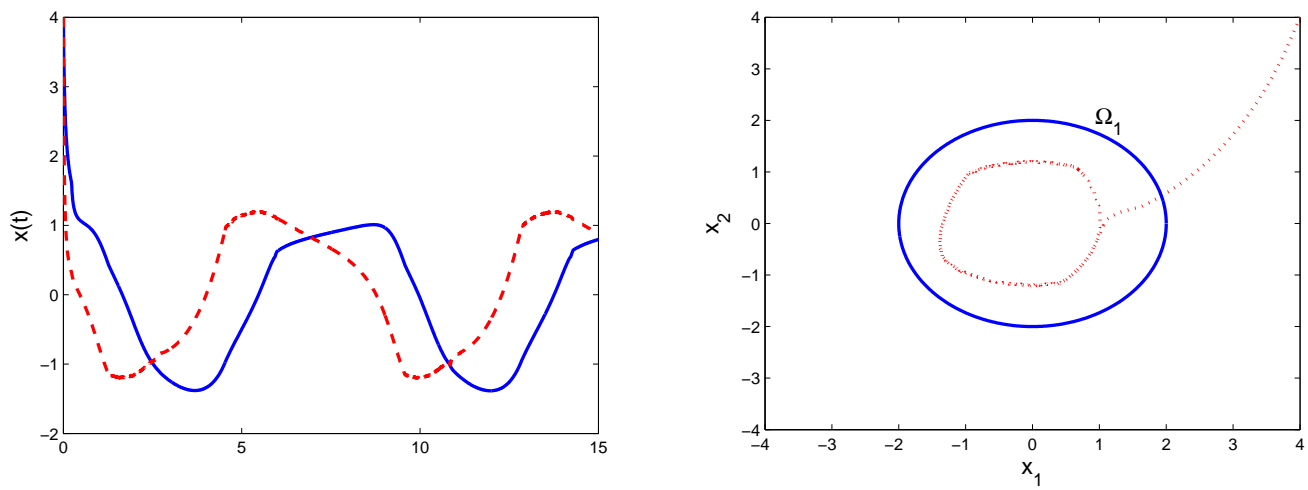

Figura 2: a) Solução chaveada para a condição inicial $x_{0}=\left[\begin{array}{l}4 \\ 2\end{array}\right]$ para o Exemplo 3.1 (b) plano de fase para o Exemplo 3.1

\section{Conclusão}

Neste artigo, o uso da matriz de Metzler permitiu resolver o problema de obter uma lei de chaveamento com múltiplas funções auxiliares para uma classe de sistemas chaveados contendo subsistemas não ultimamente limitados e, também, simplifica a obtenção da estimativa do atrator.

\section{Referências}

[1] Arrifano, N. S. D.; Oliveira, V. A.; Ramos, R. A.; Bretas S, N. G. E Oliveira, R. V., Fuzzy stabilization of power systems in a co-generation scheme subject to random abrupt variations of operating conditions, IEEE Transactions on Control System Technology v.15, n.2, pp.384-393, $200 \%$. 

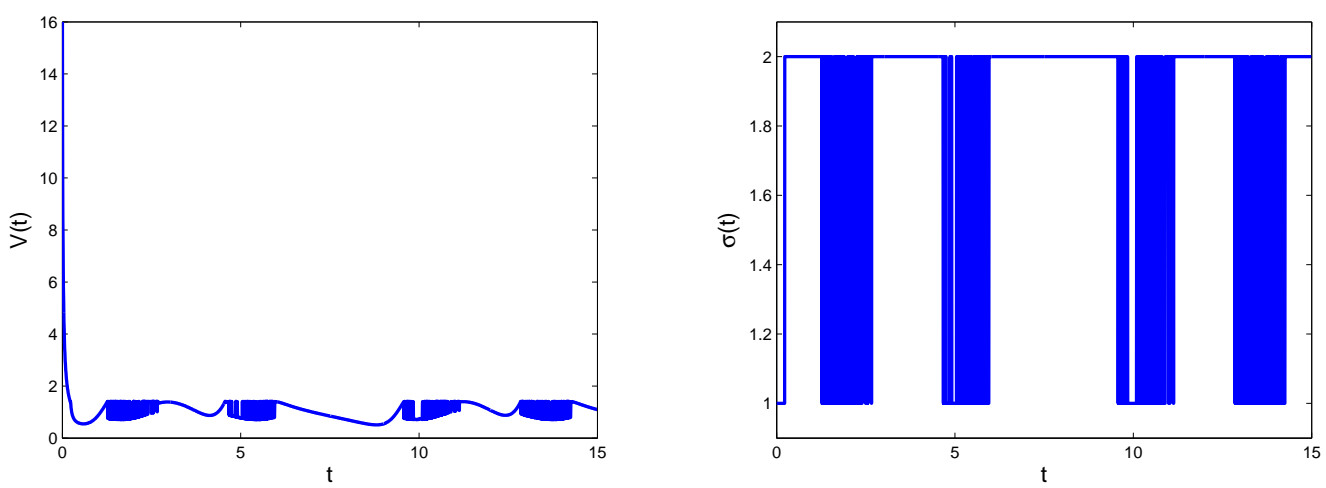

Figura 3: a) Função $V_{p}$ ao longo da solução do sistema chaveado para o Exemplo 3.1 (b) lei de chaveamento para o Exemplo 3.1

[2] A. Bacciotti e L. Mazzi, An invariance principle for nonlinear switched systems, Systems and Control Letters v.54, n.11, p.1109-1119, 2005.

[3] A. Bacciotti e L. Mazzi, From Artstein-Sontag theorem to the min-projection strategy, Transactions of the Institute of Measurement Control v.32, n.6, p.571-581, 2010.

[4] J. S. Chiou, C. J. Wang, C. M. Cheng e C. C. Wang, Analysis and synthesis of switched nonlinear systems using the T-S fuzzy model, Applied Mathematical Modelling v.34, n.6, p.1467-1481, 2010.

[5] P. Colaneri e J. C. Geromel e A. Astolfi, Stabilization of continuous-time switched nonlinear systems, Systems\& Control Letter v.5\%, n.1, p.95-103, 2008.

[6] Deaecto, G. S., "Projeto de controladores dinâmicos com comutação", Dissertação de Mestrado, Departamento de Engenharia Elétrica e de Computação, Universidade Estadual de Campinas, Campinas, Brasil, 2010.

[7] D. Liberzon, "Switching in Systems and Control", Birkhauser, Boston, USA, 2003.

[8] Valentino, M. C.; Oliveira, V. A.; Alberto, L. F. E SantAnna, D. A., An extension of the invariance principle for dwell-time switched nonlinear systems, Systems $\&$ Control Letters v.61, n.4, p.580 - 586, 2012.

[9] Michele C. Valentino, Flávio A. Faria e Vilma A. Oliveira (2013). An extension of the invariance principle for switched T-S fuzzy systems synthesis of switched nonlinear systems using the T-S fuzzy model, em "ECC013 European Control Conference" pp.4322-4327, Zrich, Switzerland, 2013.

[10] Michele C. Valentino, Flávio A. Faria e Vilma A. Oliveira (2014). Estimativa de conjuntos atratores de sistemas chaveados contínuos no tempo através de uma lei de chaveamento mista, em "CMAC-Congresso de Matemática Aplicada e Computacional", Curitiba, 2014.

[11] Michele C. Valentino (2013). Uma Extensão do Princípio de Invariância para Sistemas Chaveados, tese de Doutorado, Departamento de Engenharia Elétrica, Universidade de São Paulo, São Carlos, Brasil. 